\title{
Research on the Similarities between the Plot of Ji Chun Tai and Content of Sichuan Opera*
}

\author{
Ruoxi Li \\ Editorial Department of Journal, Sichuan University of Arts and Science, Dazhou, China
}

\begin{abstract}
Ji Chun Tai is the masterpiece of Sichuan dialect on late Qing Dynasty, composed of 40 vernacular short stories. It is divided into four parts, namely, Yuan Ji, Heng $\mathrm{Ji}$, $\mathbf{~ L i ~ J i , ~ a n d ~ Z h e n ~ J i . ~ E a c h ~ p a r t ~ c o n t a i n s ~ t e n ~}$ short stories. The author of Ji Chun Tai is a literator from Zhong Jiang who failed in imperial examination System in late Qing Dynasty. There are a large number of Sichuan Opera elements in those forty vernacular short stories. Generally speaking, the plot of $J i$ Chun Tai is full of ups and downs, together with relatively concentrated conflicts, which reflects the characteristics of Sichuan opera. Besides, the thought of persuasion and punishment, strong superstitious color, and detective story in Ji Chui Tai are combined together to reflect the characteristics of Sichuan Opera.
\end{abstract}

Index Terms—Ji Chun Tai, Sichuan opera element, thought of persuasion and punishment, detective story

\section{INTRODUCTION}

There are a large number of Sichuan dialects presented in the form of verse in Ji Chun Tai, which can be sung in songs. The elements of Sichuan opera in this novel are reflected not only in expression, but also in plot. In 2013, Li Tingting and Liu Shiren made a summary of the research on Ji Chui Tai in essay Research Status of Ji Chun Tai and Prospect in China (2013). After July 2013, researches on this novel mainly focused on three aspects: literature, culture and linguistics perspective. Researches from the perspective of Literature are Wang Yangang's Imperial Edict Preach and Colloquial Preach Novels in Qing Dynasty :A Study Based on Sichuan District (Wang,2014); Ji Yuping's A Study on The Simulating Colloquial Stories in the Ming and Qing Dynasties from the Lack of Literariness Based on Ji Chun Tai (2014 ); and Li Ruoxi's Influences on the Filial Piety Concept of Students in the Newly-authorized University from the Script Stories Printed in Sichuan in the Late Qing Dynasty: A Case Study of Ji Chun Tai (2017); Li Tingting and Liu Shiren's Filial Piety Culture Implied in Script Story Printed in Sichuan in the Late Qing Dynasty: A Case Study of Ji Chun Tai(2015).The researches from the perspective of linguistics include the master's thesis of Wang Yiyu and Li Shu:Yiyu's A Study on Dialect Vocabulary Grammar of Ji Chun Tai(2013);Li Shu's A Study of Interrogative in Ji Chun Tai(2014).So far, there are fewer studies on Ji Chun Tai from the perspective of linguistics. The research of Sichuan opera elements in the plot of $J i$ Chun Tai can not only enrich the research achievements of Sichuan dialect novel in late Qing dynasty, but also provide new ideas for future research on Sichuan opera.

\section{COMPlicated Plots ANd Concentrated CONFlicts}

The Plot of Ji Chun Tai is similar to that of detective stories in some degree, such as the first story Two Gold Bracelets in Yuan Ji(one part of Ji Chun Tai). Two Gold Bracelets tells the love story of Chang Huaide and Fang Shuying. They finally get together after suffering many hardships. They have been engaged to each other arranged by their parents since they are children. Huaide, at five years old, offended his uncle Chang Zhengtai, a cunning, presuming, opinionated and scoundrel person (Liu Xingsan, 1993, p3).Huaide hated Chang Zhengtai very much for his cruelty. When Huaide was ten years old, his father died suddenly and then his family got into financial troubles. Zhengtai deliberately made Huaide homeless, and thus Huaide and his mother had to move to the ancestral tomb guard room. Since then, Huaide's mother spun, and Huaide picked up firewood to make a living (Liu Xingsan, 1993, p3).Unfortunately, next year, Huaide's mother died. Then Zhengtai forced their relatives not to help young Huaide. Huaide had no way but to borrow money from uncle Fang. Uncle Fung was out for business, when Huaide got there. Uncle fang's daughter Shuying gave Huaide a gold bracelet with not telling him. Unfortunately, this gold bracelet was noticed by Zhengtai, who accused Huaide of stealing in order to kill him. People in the clan begged for him, but Zhengtai confiscated the gold bracelet and punished Huaide by keeping him out of their shrine forever. All their relatives were not allowed to shelter Huaide (Liu Xingsan, 1993,p 8). Therefore, Huaide became a beggar. Shuying gave Huaide another gold bracelet secretly when met him again. Huaide did not dare to mortgage it and returned it to the ancestral hall. Unfortunately, he was seen by Zhengtai at that moment. Zhengtai and Uncle Fang sent Huaide to the

\footnotetext{
* Sponsored by: Self-financing project of Sichuan education department in 2016: The Study on the Influence of Sichuan Vernacular Novels on Filial Piety of Newly-Built Undergraduates-Based on Ji Chun Tai (Project number: 165B0227) as well as the project of Sichuan University of Arts and Science in 2014: Research on the Economic and Cultural Development Pattern and Internal Mechanism of the Local Served by Featured Majors of Sichuan University of Arts and Science. (Project number: 2014XB001Y).
} 
local government with the intention of killing him. In prison, officials were impressed by Huaide and assisted him in escaping from prison secretly. Later, Huaide became famous and returned home with honor. And then, he married Shuying out of her help.

The plot of traditional Sichuan opera The Love Story of He Zhu is similar to that of Two Gold Bracelets. This novel was about a ministry councillor named Jing Sanguan who despised the poor and flattered the rich. Sanguan and his servant Wang Xing worked together to break off his daughter's engagement with Zhao Peng, but Sanguan's maid, He Zhu, knew that by accident. He Zhu did not want Zhao Peng to be homeless, so she and another servant Zhao Wang decided to give money to Zhao Peng in the name of their miss. Zhao Peng thought it was the gift from the miss and gave his handkerchief to miss in return as promise, however, that handkerchief was found by Wang Xing and the ministry councillor, and then they accused Zhao Peng of stealing. Therefore, Zhao Peng was drove out of the house, and the miss jumped into water to commit suicide. The Jing's houses were caught in a fire, and then the family went broke. Wang Xing was planning to sell He Zhu to brothel. Fortunately, Zhao Wang had a chance to rescue He Zhu from the mansion, and she chose to become a nun in the nunnery. Zhao Peng was desperate to kill himself. Luckily, he was saved by Zhao Wang who just returned from getting rent, and Zhao Wang gave that money to Zhao Peng. Eventually, Zhao Peng made up his mind to take imperial examination in Beijing and ended up being an officer Later, Zhao Wang knew that He Zhu pretended to be the miss to marry Zhao Peng. He asked Jin Sanguan to confirm the miss's identity. When the real miss returned, she blamed He Zhu, and Zhao Wang begged to miss for her leniency. Finally, Zhao Peng married those two ladies, and Jing also took Zhao Wang as his son (The Love Story of He Zhu, 2017).

The plot of Two Gold Bracelets is as complicated as that of The Love Story of He Zhu. Generally speaking, there are forty short stories in Ji Chun Tai, all of which are full of concentrated conflicts. Lu Xun introduced those stories in this way: "the story scripts by literator in Song dynasty are both plain and lackluster, and these colloquial stories tell something of the past instead of nowadays, which makes those stories far from being original. However, there is another kind of art form narrated by people from lower class, which is called as "vernacular fiction" (2006,p10).At first, vernacular fictions were created with the reference to the scripts in Wazi of Song Dynasty, and those fictions were ready to perform on stage. Later, although those vernacular fictions had been changed from their original form, but they still kept their complicated plots and concentrated contradictions. These features did not reach their climax until in Li Yu's novels. Li Yu thought vernacular fiction was silent drama, and put forward the theory of silent play (Hu Du, 1987, p11 ). Ji Chun Tai is without exception, even as the masterpiece in the decline period of vernacular fiction (Ji Yuping, 2014,p). Every story of Ji Chun Tai is similar to detective story. Each story is with long time span and complicated plot, such as Sell Mud Pill, Coincidence Marriage, Coincidence Retribution, The Love Story of Xiang Lian(Liu Xingsan,1993,p 557-565 ). What's more, these stories can stimulate the reader's interest when they read the beginning part. As the reading deepens, readers are deeply attracted by those stories, and even try to sympathize with protagonist's fortune. In the future, when they recall the plot of those stories, those characters will go to their minds naturally. Forty stories of $J i$ Chun Tai are all suitable for stage play, for their complicated plots and concentrated conflicts. Ji Chun Tai is similar to Mei Jiang-Xue which belongs to Jie Ban drama in Sichuan opera for the similarities in their plots. (Feng Shudan,1992, p 12).

\section{ENLIGHTENMENT FUNCTION}

Entertainment is the essence of drama, and education is the soul of drama, therefore education is the purpose, and entertainment is the effect (Feng Shudang, 1992, p 1).It can be said that there are a lot of dramas in Sichuan opera focused on promoting filial piety culture, so as to realize the purpose of persuasion and punishment, such as The last four books of Sichuan opera, namely The Story of the Thorns Hairpin, Three Filial Stories. Another example is the high-pitched The Great Filial Piety in traditional repertoire of Sichuan opera, namely, Three Filial Stories, Pan-zhen Recognized His Mother, The Soup Made by Filial Women (Hu Du, 1987). At the same time, the enlightenment purpose of Ji Chun Tai is also obvious, which has a lot to do with the author's personal experience. It is said that Liu Xingsan, the name of the author of $J i$ Chun Tai, turns out to be the author's pseudonym. From Emperor Tongzhi's to Guangxu's reign in Qing Dynasty, Liu Xingsan was not found in the historical records of Zhong Jiang such as County Annals of Zhong Jiang and The History of Shu. In The Preface of New Version Ji Chun Tai, Lin Youren says that Liu Xingsan of Zhong Yi is a recluse (Liu Xingsan, 1993). Although it is uncertain of the real name of the author of Ji Chun Tai, it is obvious that he is a literati failed in imperial examination. In the preface of the version edited by Cai Dongyong, there is a saying that the author participated in the imperial examination system, but failed, or maybe he got to know the official corruption so he decided to be a hermit eventually (Liu Xingsan,1993,p1).In combination with the content and the way of expression of Ji Chun Tai, it can be said that the author is also a literator failed in the imperial examination of Zhong Jiang district in late Qing dynasty, because maybe Xingsan means reflecting three times a day, which comes from Confucian saying. Ji Chun Tai's is created with the purpose to praise virtue and punish vice, and all forty stories are written for that purpose, among which there are a large number of stories straightforward preaching to the good. In late Qing Dynasty, some writers of Sichuan opera were also failed literator, such as Lin from Nan Chong. In Nan Chong, there was a person named Lin failed in the provincial graduate exam. He was good at poetry and drama. He used to study plays when smoking opium, and wrote thirty plays, but he was unwilling to tell readers his real name (Feng Shudan, 1992,p 15). It can be seen that many writers of Sichuan opera are also failed literator, similar to the author of $J i$ 
Chun Tai. This may be the main reason why their works have the purpose of persuasion and punishment.

Lin Youwei in Tongshan writes the preface for Ji Chun Tai named the Preface of New Version Ji Chun Tai. Lin Youren says that Liu Xingsan in Zhong Yi is a recluse. Liu Xingsan does not go out much and only write a book called Ji Chun Tai in order to praise virtue and punish vice(Liu Sangxin, 1993, p566). The enlightening function of persuasion and punishment goes through the whole book. For example, the fifth article of Yuan Ji (a part of Ji Chun Tai) is Moral Tiger Temple which promotes filial piety and encourages people to do filial things. In addition, the sixth article of Yuan Ji called A Magical Palm promotes filial piety and justice (Liu Sangxin, 1993, p84-84). Ji Chun Tai includes 40 stories, all of which are with educational purpose, especially the preliminaries poem Adjudge A Wolf of the fifth article of Heng Ji. It holds that there are numerous beasts in human face as well as sheep in wolf's clothing, therefore, kindness must be repaid and grievance must be resolved (Liu Sangxin, 1993, p212). It is obvious to see the flaw of this book for its focus on persuasion in content and novelty in the way of expression. At the same time, the appearance of Ji Chun Tai based on praising virtue and punishing vice is the sign that the end of the vernacular novel is on the way (Ji Yuping, 2014, p36). Although the above analysis is mainly about the shortcomings of Ji Chun Tai, it also confirms its creative purpose of persuasion and punishment.

\section{Strong Superstitious COLOR}

Ji Chun Tai's strong superstitious color is closely related to its teaching purpose of persuasion and punishment, which is reflected in its depiction of karma, gratitude from animal and ghosts. Coincidence Retribution, the tenth article of Li Ji (a part of Ji Chun Tai), tells the story of people suffering from retribution for being unfilial to their parents. Chen Weiming was not filial to his parents. He got a son named Chen Guochang when he was very old, so he spoiled his son very much. While Chen Guochang was influenced by her father, turned out be unfilial, either. When Weiming and his wife were old and weak, with no money to support themselves, he asked his son for money, but the son shouted angrily, "go away! Have you ever given money to your parents before? I have a role model. Don't ask money from me". (Liu Xingsan, 1993, p 422 ) Guochang was a tailor and made some money by his job, but he splurged his money in the city. When Guochang's cousin saw that, he scolded Guochang, "If you were not filial, were you not afraid of being damned? It was too late to repent" (Liu Xingsan,1993,p423).In order to avoid the responsibility of supporting his parents, Guochang left his hometown, and eventually Weiming and his wife died of starvation. Guochang fled to Kui's mansion, and fell in love with the county magistrate's daughter Ailian. And then, they stole Alilian's father's money and eloped with each other. They were so extravagant that they soon used up all their money. Guochang got the disease of consumption and Ailian abandoned him for his disease. Later, each time she saw Guochang, she scolded him furiously not by word, but by fist (Liu Xingsan, 1993, p 426). Later, Ailian killed Guochang on purpose. While the maid of Ailian was very filial to her father, and even sold herself in order to save her father. She was arranged by the county magistrate to marry Ailian's Finance, and they lived happily after getting married. These stories hold that there is a natural cause and effect relationship among good, evil and karma. Meanwhile, these stories use the effect of karma to educate people, in order to achieve the purpose of persuasion and punishment.

There are also many traditional dramas in Sichuan opera with strong superstitious colors, such as Four Zhu in Five Robes and Four Zhu, namely Zhou Tian Zhu, Jiu Long Zhu, Wu Xing Zhu and Qing Tian Zhu respectively. Their superstitious colors are similar to that in Ji Chun Tai. The Sichuan opera, existing at the same time as Ji Chun Tai, is mainly in the form of Mu-lian Opera. From Guang Xu's reign to the revolution of 1911, it can be said that Sichuan opera is the era of The Mu-lian Opera (Feng Shudan,1992, p46 ).The Mu-lian Opera is a kind of ancient drama which is preserved in folk activities, with the theme of the religious story Mu lian saved her Mother. It is the first recorded drama, and is known as the ancestor of Chinese opera (Mu-lian Opera, 2017).The Mu-lian Opera persuades people to be good, and it implies that if one family keep on doing good deeds, his offspring will live longer than him (Feng Shudan, 1992, p44 ). Later, the playwright Xu fen rewrites the novel Mu-lian's Mother in a new way. The story of ghost shows the affection between mother and child, and the true love of the world. With humor, vivid language, it displays distinctive characteristics of Sichuan culture. Whether karma of Ji Chun Tai or odd power of gods or ghosts in Sichuan opera, they are depicted to achieve the goal of promoting virtues and punishing evils.

\section{Highlight the Plot of Detective Story}

Ancient Chinese detective stories appeared in Song dynasty. In The general theory of Chinese fiction, it is said that this subject is a quite important one In the books of Song Dynasty, and popular with the public (Ning Zhongyi, 1995, p527). In Song dynasty, detective stories appeared with the emergence of Wazi, a kind of entertainment for lower class. Due to its compact structure and complicated plot, detective story attracts readers as soon as they read it. With the development of colloquial stories, writers at that time learn from detective story, and they tries to narrate stories in colloquial style called Ji Chun Tai. There are forty stories with the elements of detective story, and the most common case is homicide case. For example, Ten-year-old Cock, the second story of Yuan Ji (a part of Ji Chun Tai.), and Zhuo Nan Feng, the first story of Heng Ji (a part of Ji Chun Tai.) and so on (Liu Xingsan, 1993, p 470-481). These stories not only have the elements the detective story, but also with the plot from grievance to relief. They are with long time span and compact structure, which are similar to the detective story in Sichuan opera. 
In Sichuan opera, there are a lot of songs concerning the plot of detective story, like Case Opera. In Sichuan opera repertory, the proportion of traditional drama accounts for $70 \%$. The traditional opera generally refers to the repertory of the play preserved before the founding of The Republic of China. Sichuan Opera is particularly rich in classical repertory. For long time, the representative dramas of Sichuan opera are Three Kingdom, Nations Drama, Shui Hu Drama, East Window Drama, Liao Zhai Drama, Gods Drama, Public Case Drama and Five Pao,Four Zhu, Tian Yu Hua, Four books of high tune, Four books of Crokinole, Eighteen books of Rune Scape and so on. They show that the traditional plays of Sichuan opera relying on vocal singing style are mainly based on folk stories and historical novels (Zhou Qixun, 1998, p23-26). Specifically, the plot of The Life and Die Sortition is a detective story. During Jiajing period of Ming Dynasty, a pair of lovers, Zhang Jinsheng and Wang Yuhuan, went to a pavilion to shelter from the rain. They met He Sanlang there, and the son of He Zongbing. Sanlang tried to flirt with Yuhuan and died by accident. Zongbing asked Huang Boxian, the county magistrate, to decapitate Yuhuan. Huang Boxian could not bear to decapitate this innocent girl and release her. Huang's daughter Xiulan and the adopted daughter Qiuping worried about their father's fate, and both of them were willing to die for their father. Yuhuan was also afraid that her benefactor would suffer a lot , so she did not leave. Then three girls begged for "life and death cards" in front of the god. Finally, Xiulan got death card. On the court, He Zongbing asked officer to check the identity of prisoner, and sent Huang boxian to prison. When Boxian was executed death penalty, luckily, the inspector Hai Rui from HuGuang district made an inspection tour and then the grievance came to light. Whether in the form of novel or play, detective story can attract readers or viewers in short time so as to confirm its status in the field of novel and play. The plot of the detective story in Ji Chun Tai and that in Sichuan opera are full of ups and downs, concentrated and fascinating.

\section{CONCLUSION}

Ji Chun Tai is viewed as the representative of Sichuan dialect novel in late Qing Dynasty; however, its literary value as a novel has been questioned. Experts hold that its content extremely emphasizes on enlightenment but loses the artistic charm of literature. However, the elements of Sichuan opera in Ji Chun Tai cannot be ignored (Ji Yuping, 2014, p36-43). The plot of Ji Chun Tai is full of ups and downs, and conflicts are concentrated. It is created with the purpose to promote enlightenment thought of persuasion and punishment. Strong superstitious color and highlight of the plot of detective story are the specific elements of Sichuan opera. Because of the elements of Sichuan opera in the plot of $J i$ Chun Tai, more than forty short stories of it can be adapted into wonderful Sichuan opera.

\section{REFERENCES}

[1] Feng Shudan. (1992). Several Nameless Authors in The Late Qing Dynasty and the Early Republic of China. Chengdu: Shuang Qing Printing Press.

[2] Feng Shudan. (1992). Introduction of Chinese Opera with Board. Chengdu: Shuang Qing Printing Press.

[3] Feng Shudan. (1992). The Three Original Forms of Sichuan Opera. Chengdu: Shuang Qing Printing Press.

[4] Feng Shudan. (1992). Mu-lian Legend in Sichuan Opera. Chengdu: Shuang Qing Printing Press.

[5] Fu ChengZhou.(2009). LiYu's Silent Drama Theory and the Theatrical Character of Huaben. Journal of Shenzhen University (Humanities \& Social Sciences), 107-111, issue 1.

[6] Hu Du,etc.(1987). Sichuan Opera Dictionary. Beijing: China Drama Press.

[7] https://baike.baidu.com/item/\%E7\%9B\%AE\%E8\%BF\%9E\%E6\%88\%8F/9125024?fr=aladdin. (Accessed 12/25/2017)

[8] Ji Yuping. (2014). A Study on The Simulating Colloquial Stories in the Ming and Qing Dynasties from the Lack of Literariness Based on Ji Chun Tai. Journal of Southwest Minzu University (Humanities and Social Science), 180-183, issue 11.

[9] Ji Yuping. (2014). On Different Presence of The Reality--Comparing the One Hundred Years of Solitude and Life and Death are Wearing Me Out. Journal of Yancheng Institute of Technology (Social Science Edition), 36-39, issue 4.

[10] Li Ruoxi. (2017). Influences on the Filial Piety Concept of Students in the Newly-authorized University from the Script Stories Printed in Sichuan in the Late Qing Dynasty: A Case Study of Ji Chun Tai. Sichuan University Of Arts and Science Journal, $137-140$, issue 4.

[11] Li Tingting,Liu Shiren. (2013). Research Status of Ji Chun Tai and Prospect in China. Sichuan University of Arts and Science Journal, 81-85, issue 4.

[12] Li Tingting,Liu Shiren.(2015). Filial Piety Culture Implied in Script Story Printed in Sichuan in the Late Qing Dynasty:A Case Study of Ji Chun Tai. Sichuan University of Arts and Science Journal, 103-106, issue 3.

[13] Li Shuyu. (2014). A Study of Interrogative in Ji Chun Tai. Chengdu: Master Thesis of Sichuan Normal University.

[14] Liu Xingsan. (1993). Ji Chun Tai. Punctuate and Proofread by Cai Dunyong. Nanjing: Jiangsu Ancient Book Publishing House.

[15] Lu Xun. (2006). Hua Ben in Song Dynasty. Beijing: Chinese people's Literature Press.

[16] Ning Zongyi. (1995). An Introduction to Chinese Novel Studies. Hefei: Anhui Education Publishing House.

[17] The Love Story of He Zhu. http://www.chuiyue.com/eg/25235/player-0-3.html. (Accessed 12/16/2017).

[18] Wang Yangang. (2014). Imperial Edict Preach and Colloquial Preach Novels in Qing Dynasty: A Study Based on Sichuan District. Literary Heritage, 2014, issue 6.

[19] Zhou Qixu. (1998). Overview of Sichuan Opera Repertoire .Sichuan Drama, 23-26,issue 4. 
Ruoxi Li was born in Jilin, China in 1981. She received her master degree in Literature from Ningxia University, China in 2011. She is currently a research assistant of Journal Editorial Department, Sichuan University of Arts and Science, Sichuan, China. Her research interests are ancient Chinese literature. 